\title{
ARTICLE OPEN \\ Non-stoquastic Hamiltonians in quantum annealing via geometric phases
}

Walter Vinci ${ }^{1,2,3}$ and Daniel A. Lidar ${ }^{1,2,3,4}$

We argue that a complete description of quantum annealing implemented with continuous variables must take into account the non-adiabatic Aharonov-Anandan geometric phase that arises when the system Hamiltonian changes during the anneal. We show that this geometric effect leads to the appearance of non-stoquasticity in the effective quantum Ising Hamiltonians that are typically used to describe quantum annealing with flux qubits. We explicitly demonstrate the effect of this geometric nonstoquasticity when quantum annealing is performed with a system of one and two coupled flux qubits. The realization of nonstoquastic Hamiltonians has important implications from a computational complexity perspective, since it is believed that in many cases quantum annealing with stoquastic Hamiltonians can be efficiently simulated via classical algorithms such as Quantum Monte Carlo. It is well known that the direct implementation of non-stoquastic Hamiltonians with flux qubits is particularly challenging. Our results suggest an alternative path for the implementation of non-stoquasticity via geometric phases that can be exploited for computational purposes.

npj Quantum Information (2017)3:38; doi:10.1038/s41534-017-0037-z

\section{INTRODUCTION}

It is well known that the solution of computational problems can be encoded into the ground state of a time-dependent quantum Hamiltonian. This approach is known as adiabatic quantum computation (AQC), ${ }^{1-3}$ and is universal for quantum computing ${ }^{4}$ (for a review of $A Q C$, see ref. 5). Quantum annealing (QA) is a framework that incorporates algorithms ${ }^{6-8}$ and hardware $e^{9-13}$ designed to solve computational problems via quantum evolution toward the ground states of final Hamiltonians that encode classical optimization problems, without necessarily insisting on universality or adiabaticity.

QA thus inhabits a regime that is intermediate between the idealized assumptions of universal AQC and unavoidable experimental compromises. Perhaps the most significant of these compromises has been the design of stoquastic quantum annealers. A Hamiltonian $H$ is stoquastic with respect to a given basis if $H$ has only real nonpositive off-diagonal matrix elements in that basis. This means that its ground state can be expressed as a classical probability distribution, ${ }^{14,}{ }^{15}$ and that the standard quantum-to-classical mapping ${ }^{16}$ used in quantum Monte Carlo algorithms does not result in a "sign problem" (a partition function with Boltzmann weights taking positive as well as unphysical negative, or even complex values). ${ }^{14,15,17-21}$ The computational power of stoquastic Hamiltonians has been carefully scrutinized, and is suspected to be limited in the ground-state AQC setting. ${ }^{5}$ For example, it is unlikely that ground-state stoquastic $A Q C$ is universal. ${ }^{22}$ In many cases ground-state stoquastic AQC can be efficiently simulated by classical algorithms, but certain exceptions are known. ${ }^{23,24}$

One is thus naturally motivated to consider a departure from the stoquastic setting. Indeed, this is the setting of proofs of the universality of AQC and of various specific results that use nonstoquasticity to improve upon the performance of a stoquastic Hamiltonian. ${ }^{25-31}$ For example, it is known that non-stoquastic interactions that are turned on temporarily during QA can modify the annealing path so that it encounters a polynomially small gap rather than an exponentially small one, by replacing a first-order quantum phase transition by a second-order one. ${ }^{31}$ Note that (non-)stoquasticity is a global property of the entire Hamiltonian (since once can always change the stoquasticity of a local term by a local change of basis), and henceforth when we-with slight abuse of language-refer to (non-)stoquastic interactions (or terms) we have in mind a fixed basis. Motivated by experimental considerations, we shall focus on the computational basis, i.e., the basis in which the final Hamiltonian is diagonal.

Introducing non-stoquastic interactions is especially important in the physical implementation of QA devices, in order to allow them to escape the trap of efficient classical simulability. The DWave devices, ${ }^{10-12}$ e.g., can only implement the Hamiltonian of the quantum transverse field Ising model, which is stoquastic. To remedy this would require additional couplings between the flux qubits, to realize, e.g., $\sigma^{x} \otimes \sigma^{x}$ interactions, in addition to the existing $\sigma^{z} \otimes \sigma^{z}$ interactions. However, the implementation of a scalable architecture with such interactions is highly demanding, at least with superconducting flux qubits.

Rather than attempt to introduce non-stoquasticity via new components, here we revisit the assumptions that lead to the derivation of the Hamiltonian generating the effective time evolution of a continuous-variable system, such as inductively coupled flux qubits. We show that non-stoquastic terms arise naturally as a non-adiabatic geometric phase, due to the Aharonov-Anandan effect, ${ }^{32,33}$ when QA is performed in systems

\footnotetext{
${ }^{1}$ Department of Electrical Engineering, University of Southern California, Los Angeles, CA 90089, USA; ${ }^{2}$ Department of Physics and Astronomy, University of Southern California,

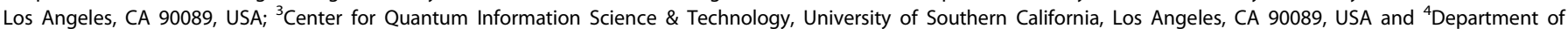
Chemistry, University of Southern California, Los Angeles, CA 90089, USA

Correspondence: Walter Vinci (wltvinci@gmail.com)
}

Received: 31 March 2017 Revised: 24 July 2017 Accepted: 31 July 2017

Published online: 21 September 2017 
of inductively coupled flux qubits. The geometric effect discussed here is distinct from earlier work on holonomic quantum computation, ${ }^{34-36}$ and non-adiabatic geometric phases in quantum computation using Josephson devices. ${ }^{37,} 38$

We study these geometric terms in detail, and show that the geometric effect is amplified in proportion to the inverse gap of the flux Hamiltonian, appearing and then disappearing during the anneal. The geometric effect can thus be considered as a type of non-stoquastic catalyst, ${ }^{5}$ with the potential to lead to quantum speedups. ${ }^{25-31}$ From a more general experimental perspective, the presence of the geometric terms studied in this paper has clear signatures that must be taken into account in the validation and characterization of current and future QA devices.

\section{RESULTS}

General formalism and the geometric term

For concreteness, we assume that QA is performed by implementing a time-dependent Hamiltonian that can be generically expressed as follows:

$H(\phi, t)=-\frac{1}{2} E_{C} \sum_{i=1}^{n} \partial_{\phi_{i}}^{2}+P(\phi, t)$.

Such Hamiltonians can be realized with superconducting flux qubits, $^{39}$ in which case the continuous variables $\phi=\left\{\phi_{i}\right\}_{i=1}^{n}$ are the magnetic fluxes trapped by the $n$ flux qubits, and $E_{C}$ represents a charging energy. The term $P(\phi, t)$ is a timedependent potential that controls both the anneal and the interactions between qubits. We assume that the lowest $2^{n}$ energy levels of the Hamiltonian (1) are separated from the rest of the spectrum by an energy gap. For a slowly variable potential $P(\phi, t)$, the system of Eq. (1) is then confined to an $N=2^{n}$ dimensional Hilbert space $\mathcal{H}_{N}(t)$.

To proceed, we follow the approach introduced by Anandan in the discussion of non-adiabatic non-Abelian geometric phases. ${ }^{33}$ We first consider the time-evolved $N$-dimensional subspace $\mathcal{H}_{N}(t)$, which is defined by the map

$U(t): \mathcal{H}_{N}(0) \mapsto \mathcal{H}_{N}(t)=U(t) \mathcal{H}_{N}(0)$,

where $U(t)=\mathcal{T} \exp \left(-i \int_{0}^{t} H\left(t^{\prime}\right) d t^{\prime}\right)$ is the unitary time-evolution operator $(\mathcal{T}$ denotes time ordering) and $H(t)$ is the Hamiltonian (1). Let $\left\{\left|\psi_{a}(0)\right\rangle\right\}_{a=1}^{N}$ denote an orthonormal basis for $\mathcal{H}_{N}(0)$. Thus, $\mathcal{H}_{N}(t)$ has a basis whose orthonormal elements obey the Schrödinger equation:

$i \partial_{t}\left|\psi_{a}(t)\right\rangle=H(t)\left|\psi_{a}(t)\right\rangle$.

We now define another (arbitrary) orthonormal basis $\left\langle\psi_{a}^{\prime}(t)\right\rangle$ for $\mathcal{H}_{N}(t)$, such that $\left|\psi_{a}(0)\right\rangle=\left|\psi_{a}^{\prime}(0)\right\rangle$. Then there exists an $N \times N$ unitary transformation $W(t)$ between the two bases such that $\left|\psi_{b}(t)\right\rangle=\sum_{a=1}^{N} W_{a b}(t)\left|\psi_{a}^{\prime}(t)\right\rangle$. An arbitrary state $|\omega(0)\rangle=$ $\sum_{a} \omega_{a}(0)\left|\psi_{a}(0)\right\rangle \in \mathcal{H}_{N}(0)$ thus evolves according to: $|\omega(t)\rangle=$ $\sum_{a}^{a} \omega_{a}(0)\left|\psi_{a}(t)\right\rangle=\sum_{a} \omega_{a}(t)\left|\psi_{a}^{\prime}(t)\right\rangle$, where $\omega_{a}(t) \equiv \sum_{b} W_{a b}(t)$ $\omega_{b}(0)$. The unitary $W(t)$ can thus be considered as describing the effective evolution of the state $|\omega(t)\rangle$ inside the subspace $\mathcal{H}_{N}(t)$ in the frame rotating with the basis $\left|\psi_{a}^{\prime}(t)\right\rangle$ (see $S M$, section $\mathrm{A}$, for more details). By substituting the basis transformation into Eq. (3) one easily finds:

$i \partial_{t} W(t)=H^{\mathrm{eff}}(t) W(t), \quad H^{\mathrm{eff}}(t) \equiv \tilde{H}(t)-G(t)$,

where $\tilde{H}(t)$ and $G(t)$ are the $N \times N$ matrices defined by the following matrix elements, respectively:

$$
\begin{aligned}
& \tilde{H}_{a b}(t) \equiv\left\langle\psi_{a}^{\prime}(t)|H(t)| \psi_{b}^{\prime}(t)\right\rangle, \\
& G_{a b}(t) \equiv\left\langle\psi_{a}^{\prime}(t)\left|i \partial_{t}\right| \psi_{b}^{\prime}(t)\right\rangle .
\end{aligned}
$$

Let us now assume that $H(t)$ depends on $t$ only via the invertible and differentiable "annealing schedule" $s \equiv K^{-1}(\tau)$, where $\tau \equiv t / t_{f}$, and $t_{f}$ denotes the final time. Then it follows directly from Eq. (5b) that $G(t) d t=G(s) d s$ (see $S M$, section B), which shows that $G(s)$ is a geometric term, i.e., it depends only on the schedule $s$ (and not on its parametrization). ${ }^{40,41}$ Consequently, $W\left(t_{f}\right)=$ $\mathcal{T} \exp \left[-i \int_{0}^{1} H^{\text {eff }}(s) d s\right]$ with the dimensionless effective Hamilto-
nian

$H^{\mathrm{eff}}(s)=t_{f} \dot{K}(s) \tilde{H}(s)-G(s)$,

where from hereon a dot denotes $d / d s$, and we set $s \equiv \tau$ for simplicity. Note that the geometric term is negligible only in the adiabatic limit $t_{f} \rightarrow \infty$.

Application to QA with superconducting flux qubits Let $\{|a(s)\rangle\}$, with $a=1, \ldots, N$, denote the $N$ lowest instantaneous energy eigenstates of the original Hamiltonian (1), i.e., $H(s)|a(s)\rangle=E_{a}(s)|a(s)\rangle$. We identify the earlier $\left\{\left|\psi_{a}^{\prime}(s)\right\rangle\right\}$ with these eigenstates, so that $W(s)$ describes the unitary evolution in the subspace rotating with the instantaneous eigenbasis of $H(s)$. In this basis, the QA process is described by the dimensionless effective Hamiltonian (6) with:

$\tilde{H}_{a b}(s) \equiv E_{a}(s) \delta_{a b}, \quad G_{a b}(s) \equiv\left\langle a(s)\left|i \partial_{s}\right| b(s)\right\rangle$,

in which $|b(s)\rangle$, with $b=1, \ldots, N$, also labels the $N$ lowest instantaneous energy eigenstates. In QA applications, the Hamiltonian (1) is designed such that its $N=2^{n}$ lowest energy levels can be put into 1-to-1 correspondence with the energy levels of a transverse field Ising model with $n$ qubits. Thus, there exists a unitary $V(s)$ such that, up to a term proportional to the identity matrix ${ }^{42}$ :

$V(s) \tilde{H}(s) V^{\dagger}(s)=A(s) \tilde{H}^{X}+B(s) \tilde{H}^{Z}$,

where the profile functions $A(s)$ and $B(s)$ are particular to the qubit Hamiltonian (see $\mathrm{SM}$, section $\mathrm{C}$ ), $\tilde{H}^{X}=-\sum_{i=1}^{n} \sigma_{i}^{X}$ is the usual transverse field driver, and

$\tilde{H}^{z}=\sum_{i=1}^{n} h_{i} \sigma_{i}^{z}+\sum_{i>j}^{n} J_{i j} \sigma_{i}^{z} \sigma_{j}^{z}$

is the problem Hamiltonian whose ground state encodes the answer to the optimization problem of interest. The unitary $V(s)$ is the transformation between the energy eigenbasis and the computational basis $\left|\psi_{a}^{\complement}(s)\right\rangle$. Note that the geometric term transforms as a geometric connection ${ }^{33}$ (see SM, section D):

$G^{\mathrm{C}}(s)=V(s) G(s) V^{\dagger}(s)+i V(s) \dot{V}^{\dagger}(s)$.

QA of a system of $n$ flux qubits controlled by the Hamiltonian (1) is then described by the following effective Hamiltonian in the computational basis:

$H^{\text {eff, },}(s)=t_{f}\left(A(s) \tilde{H}^{X}+B(s) \tilde{H}^{Z}\right)-G^{\mathrm{C}}(s)$.

The first term, proportional to $t_{f}$ is the usual Hamiltonian discussed in literature in the context of QA. The second term has a geometric origin and is non-vanishing for finite annealing times $t_{f}$. The geometric term is non-stoquastic. To see this, note that the original Hamiltonian (1) is real, and thus there is a basis choice in which the energy eigenbasis states $|a(s)\rangle$ have only real amplitudes. Consequently, the geometric term G(s) in Eq. (7) is then a purely imaginary Hermitian matrix. The transformed geometric term $G^{C}(s)$ (Eq. (10)) is also purely imaginary in the computational basis, since the basis change of Eq. (8) can be performed with a real unitary (i.e., orthogonal) matrix $V(s)$. Therefore, including only interactions up to two-body terms, the geometric term can be written in the most general form as follows:

$G^{C}(s)=\sum_{i} g_{i}^{y}(s) \sigma_{i}^{y}+\sum_{i \neq j} g_{i j}^{x y}(s) \sigma_{i}^{x} \sigma_{j}^{y}+g_{i j}^{z y}(s) \sigma_{i}^{z} \sigma_{j}^{y}$. 
QA with geometric terms: one qubit

We now apply the results obtained so far to QA with one qubit. For concreteness we focus on the C-shunt flux qubit with three Josephson junctions. This qubit can be described by the following Hamiltonian ${ }^{43-45}$ (see SM, section E):

$H_{1}(\phi, s)=-\frac{1}{8} E_{\varsigma} \partial_{\phi}^{2}+P(\phi, s)$

$P(\phi, s)=-2 E_{J}\left(\cos \left[\frac{1}{2} \phi_{C J}^{x}(s)\right] \cos \left[\phi^{x}(s)+2 \phi\right]+\cos \phi\right)$,

where $\phi$ is the flux (in units of $\Phi_{0} / 2 \pi$ ) trapped in the superconducting ring, $E_{\mathrm{S}}$ is the charging energy of the shunting capacitor, and $\phi_{\mathrm{CJJ}}^{x}(s)$ and $\phi^{x}(s)$ are external fluxes used to control the anneal.

We next construct the effective Hamiltonian of Eq. (11). We start by numerically computing the ground state $|0(s)\rangle$ and first excited state $|1(s)\rangle$ of the flux qubit Hamiltonian Eq. (13) (examples are given in the SM, Fig. 5b, d), from which we numerically obtain the effective Hamiltonian (Eq. (6)) in the instantaneous energy eigenbasis:

$H_{1}^{\mathrm{eff}}(s)=t_{f} \frac{\Delta(s)}{2} \sigma^{z}-g(s) \sigma^{y}$,

(we have ignored a term proportional to the identity matrix) where $\Delta(s) \equiv E_{1}(s)-E_{0}(s)$ is the gap (plotted in the left panel of Fig. 1) and $g(s) \equiv\left\langle\left. 1(s)\right|_{s} \mid 0(s)\right\rangle$. We now transform to the computational basis using the unitary $V(s)=$ $\exp \left[\frac{i}{2} \arctan \left(\frac{A(s)}{B(s)}\right) \sigma^{y}\right]$, after which the Hamiltonian of Eq. (14) becomes

$H_{1}^{\mathrm{eff}, \mathrm{C}}(s)=t_{f}\left[A(s) \sigma^{x}+B(s) \sigma^{z}\right]-g^{y}(s) \sigma^{y}$.

This has the form of the most general single-qubit Hamiltonian: $H_{1}^{\text {eff,C }}(s)=\sum_{a \in x, y, z} c_{a}(s) \sigma^{a}$. It can be checked easily that (see SM,

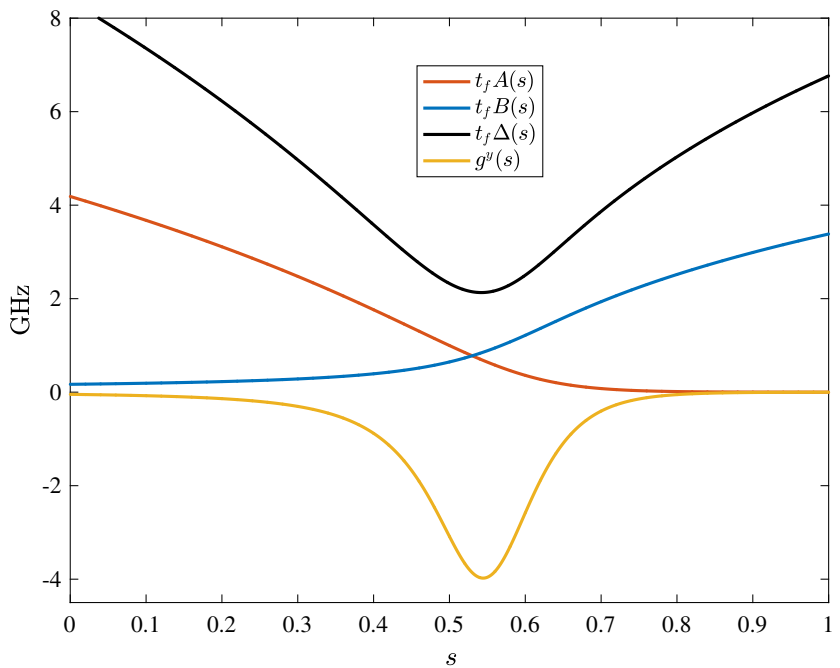

section $\mathrm{F})$ :

$\Delta(s)=2 \sqrt{A^{2}(s)+B^{2}(s)}$,

$g^{y}(s)=g(s)+\frac{2}{\Delta^{2}(s)}[\dot{A}(s) B(s)-A(s) \dot{B}(s)]$.

It is interesting to note that the second term in Eq. (16b) is similar to, but different from, the counterdiabatic term needed to obtain a "shortcut to adiabaticity"46, 47 when QA is modeled directly in terms of a spin-system Hamiltonian with a transverse field.

By defining the computational basis as the basis of states with well-defined persistent current, the annealing schedule functions $A(s)$ and $B(s)$ (shown in the left panel of Fig. 1) can be determined in terms of the external fluxes $\phi_{C j}^{x}$ and $\phi^{x}$ of Eq. (13b) (see the SM, section C). Thus, Eq. (16b) shows that these flux parameters in turn control the properties of the geometric term $g^{y}$. The profile function $g^{y}(s)$ for the geometric term, also shown in the left panel of Fig. 1, is non-vanishing toward the middle of the adiabatic evolution, when the gap closes. The effects of the geometric term are shown in the right panel of Fig. 1, obtained by numerically solving for the corresponding unitary evolution. The effects become significant when the gap is small. There is also a significant effect on the final ground-state population.

QA with geometric terms: two qubits

To study the interacting case, we consider two inductively coupled compound Josephson junction flux qubits ${ }^{39}$ (see SM, section E):

$$
\begin{aligned}
H_{2}\left(\phi_{1}, \phi_{2}, s, h_{1}, h_{2}, J_{12}\right)= & H_{1}\left(\phi_{1}, s, h_{1}\right)+ \\
& +H_{1}\left(\phi_{2}, s, h_{2}\right)+P_{\text {int }}\left(\phi_{1}, \phi_{2}, s, J_{12}\right),
\end{aligned}
$$

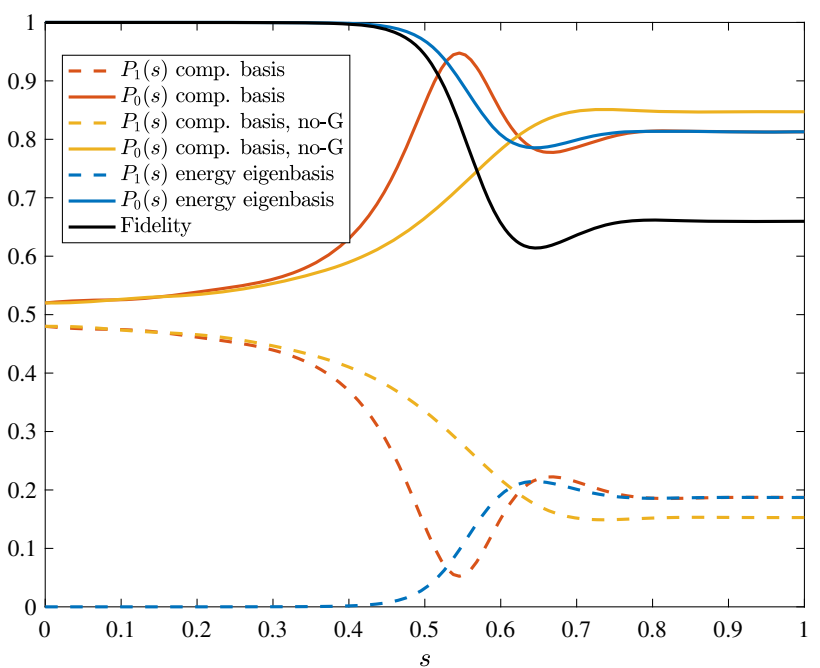

Fig. 1 One qubit. Left panel: annealing schedules $A(s)\left(\right.$ red) and $B(s)$ (blue), gap $\Delta(s)$ (black) and the geometric term $g^{y}(s)$ (yellow) of Eq. (15). The functions $A(s)$ and $B(s)$ are obtained according to the procedure described in the SM, section C. Right panel: populations of the states that are connected to the final ground state (solid) and final excited state (dashed), for $t_{f}=5(\mathrm{~ns} / 2 \pi)$. Blue and red lines represents the populations of the two states in the instantaneous energy eigenbasis (Eq. (14)) and the computational basis (Eq. (15)), respectively. Because the two Hamiltonians are equivalent up to a time-dependent basis change, and the final basis is the computational basis in both cases, the populations at the end of the evolution $(s=1)$ coincide. Yellow lines represent the populations during the anneal if the geometric term of Eq. (15) is dropped. The black line is the fidelity $\left|\left\langle\psi_{1, G}^{C}(s) \mid \psi_{1, n o-G}^{C}(s)\right\rangle\right|^{2}$ between the time-evolved states with ("G" subscript) and without ("no-G" subscript) geometric interactions. Results shown are for $\phi_{\mathrm{CJ}}^{x}(s)=2.9(1-s)+2.2 s$ and parameter values for the flux qubit Hamiltonian that match those of the highly coherent flux qubits studied in ref. $43: E_{S} / 2 \pi \hbar=3.03 \mathrm{GHz}, E_{J} / 2 \pi \hbar=86.2 \mathrm{GHz}$ (the control fluxes $\phi_{\mathrm{CJ}}^{x}(s)$ and $\phi^{x}(s)$ are shown in the SM, Fig. 2a) 
where the flux qubit Hamiltonian is given by

$H_{1}\left(\phi, s, h_{i}\right)=-\frac{1}{4} E_{C} \partial_{\phi}^{2}+P\left(\phi, s, h_{i}\right)$

$P\left(\phi, s, h_{i}\right)=2 E_{\mathrm{J}} \cos (\phi) \cos \left[\frac{\phi_{\mathrm{CJ}}^{X}(s)}{2}\right]+E_{\mathrm{L}} \frac{\left[\phi-h_{i} \phi^{X}(s)\right]^{2}}{2}$

and the interaction potential is explicitly written as:

$P_{\text {int }}\left(\phi_{1}, \phi_{2}, s, J_{12}\right)=-J_{12} E_{\mathrm{M}}\left[\phi_{1}-\phi^{x}(s)\right]\left[\phi_{2}-\phi^{x}(s)\right]$.

Proceeding as in the single-qubit case, we start from the Hamiltonian (17) to numerically compute $\tilde{H}$ and $G$ appearing in Eq. (7), and construct the effective Hamiltonian in the instantaneous energy eigenbasis (Eq. (6)). Once again, the effective Hamiltonian can be expressed in the computational basis by (numerically) finding a unitary $V$ such that the final Hamiltonian reads:

$$
\begin{gathered}
H_{2}^{\mathrm{eff}, \mathrm{C}}\left(s, h_{1}, h_{2}, J_{12}\right)=t_{f} A(s)\left(\sigma_{1}^{x}+\sigma_{2}^{x}\right)+ \\
+t_{f} B(s)\left(h_{1} \sigma_{1}^{z}+h_{2} \sigma_{2}^{z}+J_{12} \sigma_{1}^{z} \sigma_{2}^{z}\right)-G^{C}\left(s, h_{1}, h_{2}, J_{12}\right) .
\end{gathered}
$$

This is the usual transverse field Ising model, plus an additional geometric term, whose general form is given in Eq. (12). The left panel of Fig. 2 shows the gaps $\Delta_{k, 0}=E_{\mathrm{k}}-E_{0}$, where $\left\{E_{k}\right\}_{k=0}^{3}$ are the ordered eigenvalues of $H_{2}^{\text {eff, }}$, and the components of the geometric term $G^{\mathrm{C}}\left(s, h_{1}, h_{2}, J_{12}\right)$ in the computational basis as defined in Eq. (12). The left panel of Fig. 2 shows that, in general, the geometric functions $g_{i}^{y}(s), g_{i j}^{x y}(s), g_{i j}^{z y}(s)$ are all non-vanishing, and their magnitude grows as the ground-state gap shrinks. In particular, as in the single-qubit case, geometric effects introduce a non-stoquastic contribution to the driver term which is nonvanishing toward the middle of the anneal. As in the single-qubit case, the right panel of Fig. 2 shows that ignoring the geometric terms results in consistently different final populations in the computational basis.

Dependence on the annealing time

The contribution of the geometric terms is inherently a nonadiabatic effect, arising when diabatic transitions populate the excited states. In Fig. 3 we show the fidelity $\left|\left\langle\psi_{1, G}^{C}(s) \mid \psi_{1, \text { no-G }}^{C}(s)\right\rangle\right|^{2}$ between the time-evolved states with and without geometric terms, as a function of the total annealing time. As expected, the effect of the geometric terms increases with decreasing annealing time and it is reflected in the decreasing fidelity. Figure 3 shows that the total annealing time over which this contribution is significant is on the order of a few nanoseconds, for parameters relevant for current QA devices. While this is significantly shorter than the typical microsecond timescale of current QA experiments using the D-Wave devices, this is the case for the one and twoqubit cases which we have analyzed here. Since, as is clear from Eq. (16b) and from Fig. 2, the magnitude of the geometric term grows in inverse proportion to the gap, we expect it to become more significant for multi-qubit problems whose gap dependence can be inverse polynomial or even exponential. This effect is already visible in Fig. 3, which shows that the fidelity for the two-

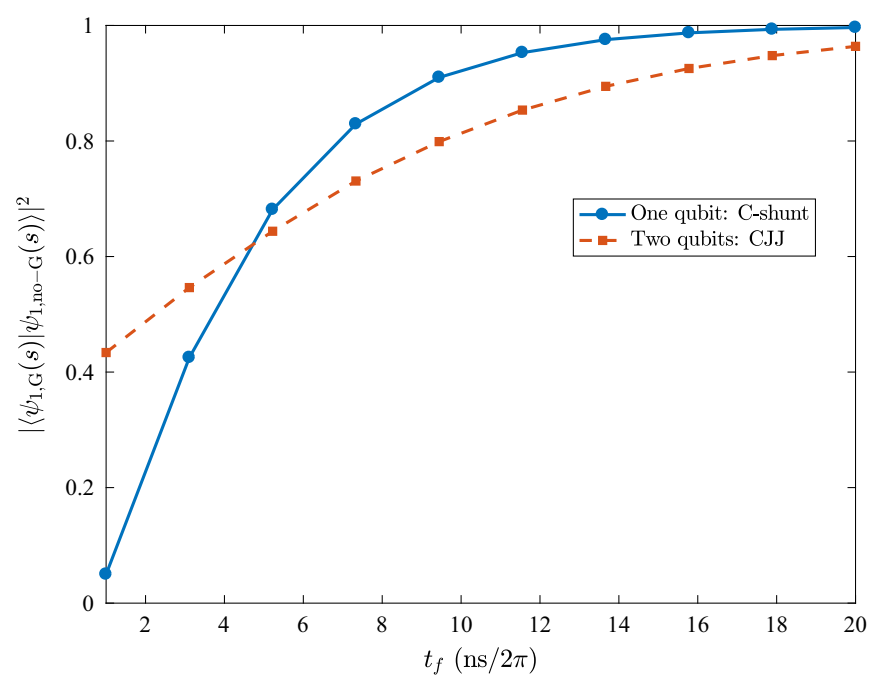

Fig. 3 Fidelity at the end of the anneal as a function of the annealing time for single qubit (blue) and two qubits (red)
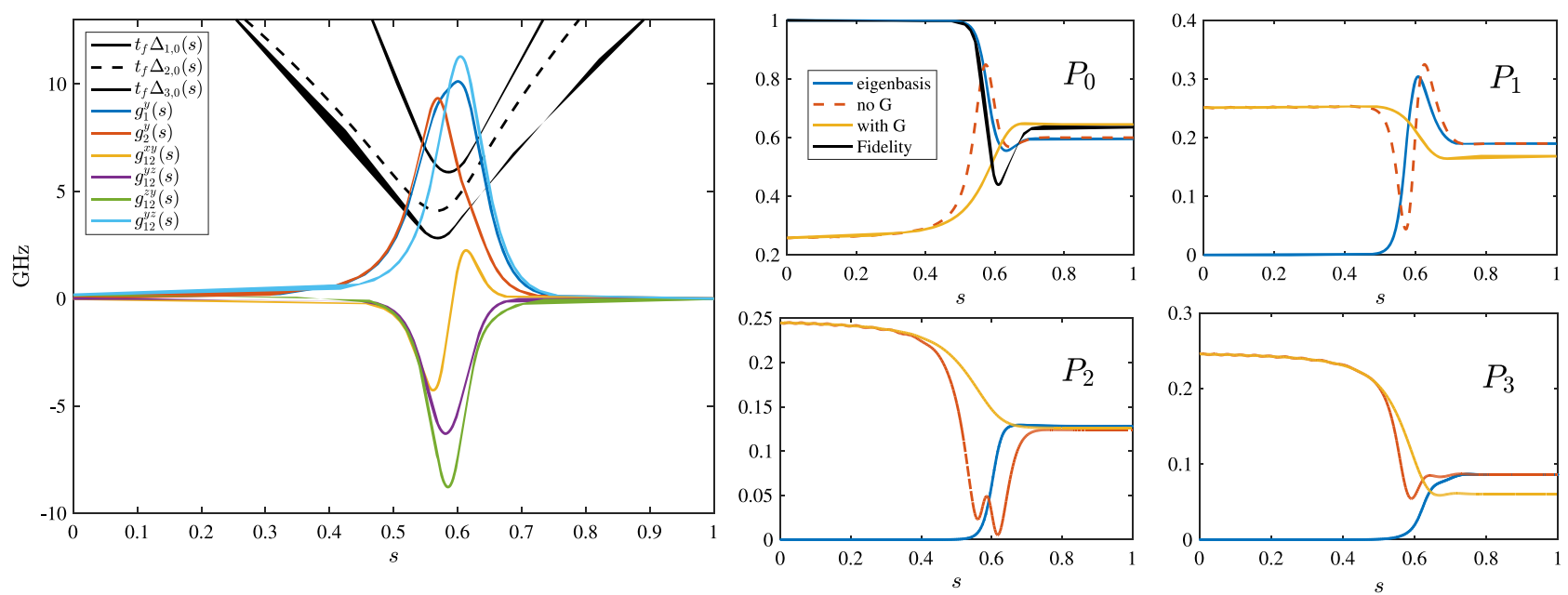

Fig. 2 Two interacting qubits. Left panel: gaps $\Delta_{k, 0}(s)$ (black) and the geometric terms $g_{i}^{y}(s), g_{i j}^{x y}(s), g_{i j}^{z y}(s)$ computed numerically using Eq. (17) for $h_{1}=1, h_{2}=0.4, J_{12}=-0.7$. Right panel: populations $P_{\mathrm{i}}$ of the states of the instantaneous basis that are connected to the final eigenstates, for the same value of the couplings as in the left panel and $t_{f}=5(\mathrm{~ns} / 2 \pi)$. The black dotted line shown in the top left panel is the fidelity as explained in Fig. 1. Results shown are for $E_{\mathrm{C}} / 2 \pi \hbar=3.44 \mathrm{GHz}, E_{\mathrm{J}} / 2 \pi \hbar=684 \mathrm{GHz}, E_{\mathrm{L}} / 2 \pi=570 \mathrm{GHz}$, and $E_{\mathrm{M}} / 2 \pi \hbar=3.98 \mathrm{GHz}$, values that are typical for the D-Wave devices ${ }^{10-12}$ (the control fluxes are shown in the SM, Fig. 1a). For consistency with the one C-shunt flux qubit case we set $\phi_{\text {CJ }}^{x}(s)=2.6(1-s)+1.9 s$ 
qubit system tends to be smaller than the one-qubit system for larger annealing times.

\section{DISCUSSION}

We have shown that even the simplest implementation of QA with flux qubits induces effective Hamiltonians with non-stoquastic interactions arising from a geometric phase. The appearance of such interactions is ubiquitous when the Hamiltonian of a continuous-variable system is changed over time, and the evolution is non-adiabatic, due to the appearance of the Aharonov-Anandan effect. Since arbitrarily small gaps are inevitable in QA for hard optimization problems, non-adiabatic evolutions are ultimately inescapable. We thus argue that, similarly, the geometric effects studied here are unavoidable and relevant in practical applications of QA. It is possible that inclusion of non-adiabatic excitations is enough to prevent the existence of efficient classical simulations of stoquastic Hamiltonians: strikingly, an example of an exponential quantum speedup is known in this case for the "glued trees" problem, ${ }^{48}$ and stoquastic $A Q C$ is universal when the computation is crafted to carefully exploit excited states. ${ }^{49}$ The inclusion of geometric effects provides an alternative and desirable mechanism to include non-stoquastic interactions in the effective Hamiltonian of $Q A$ realized with continuous-variables systems. It is likely that, in order to exploit geometric terms for computational purposes, a certain level of controllability will be required. For example, the relative strength of the geometric term can be directly controlled by modifying the annealing schedule (e.g., the function $\dot{\kappa}(s)$ in Eq. (6)) while keeping the total annealing time $t_{f}$ constant. We leave to future work the important question of whether new designs of flux qubits might enable a higher level of controllability of the geometric terms discussed here.

\section{METHODS}

For more details on the experimental and numerical methodologies employed, see Supplementary Information.

\section{Data availability}

The authors declare that the main data supporting the finding of this study are available within the article and its Supplementary Information.

\section{ACKNOWLEDGEMENTS}

We are grateful to Dr. Andrew Kerman for useful discussions. This work was supported under ARO grant number W911NF-12-1-0523, ARO MURI Grant Nos. W911NF-11-1-0268 and W911NF-15-1-0582, and NSF grant number INSPIRE-1551064. The research is based on work partially supported by the Office of the Director of National Intelligence (ODNI), Intelligence Advanced Research Projects Activity (IARPA), via the U.S. Army Research Office contract W911NF-17-C-0050. The views and conclusions contained herein are those of the authors and should not be interpreted as necessarily representing the official policies or endorsements, either expressed or implied, of the ODNI, IARPA, or the U.S. Government. The U.S. Government is authorized to reproduce and distribute reprints for Governmental purposes notwithstanding any copyright annotation thereon.

\section{AUTHOR CONTRIBUTIONS}

All authors researched, collated, and wrote this paper.

\section{ADDITIONAL INFORMATION}

Supplementary Information accompanies the paper on the npj Quantum Information website (doi:10.1038/s41534-017-0037-z).

Competing interests: The authors declare no competing financial interests.

Publisher's note: Springer Nature remains neutral with regard to jurisdictional claims in published maps and institutional affiliations.

\section{REFERENCES}

1. Farhi, E., Goldstone, J., Gutmann, S. \& Sipser, M. Quantum computation by adiabatic evolution. Preprint at http://arxiv.org/pdf/quant-ph/0001106 (2000).

2. Farhi, E. et al. A quantum adiabatic evolution algorithm applied to random instances of an NP-complete problem. Science 292, 472-475 (2001).

3. van Dam, W., Mosca, M. \& Vazirani, U. How powerful is adiabatic quantum computation? Proceedings of the 42nd IEEE Symposium on Foundations of Computer Science, 279-287 (2001).

4. Aharonov, D. et al. Adiabatic quantum computation is equivalent to standard quantum computation. SIAM J. Comput. 37, 166-194 (2007).

5. Albash, T. \& Lidar, D. A. Adiabatic quantum computing. Preprint at http://arxiv. org/pdf/quant-ph/1611.04471 (2016).

6. Kadowaki, T. \& Nishimori, H. Quantum annealing in the transverse Ising model. Phys. Rev. E 58, 5355 (1998).

7. Santoro, G. E., Martoňák, R., Tosatti, E. \& Car, R. Theory of quantum annealing of an Ising spin glass. Science 295, 2427-2430 (2002).

8. Das, A. \& Chakrabarti, B. K. Colloquium: quantum annealing and analog quantum computation. Rev. Mod. Phys. 80, 1061-1081 (2008).

9. Brooke, J., Rosenbaum, T. F. \& Aeppli, G. Tunable quantum tunnelling of magnetic domain walls. Nature 413, 610-613 (2001).

10. Johnson, M. W. et al. Quantum annealing with manufactured spins. Nature 473, 194-198 (2011).

11. Johnson, M. W. et al. A scalable control system for a superconducting adiabatic quantum optimization processor. Supercond. Sci. Technol. 23, 065004 (2010).

12. Harris, R. et al. Experimental investigation of an eight-qubit unit cell in a superconducting optimization processor. Phys. Rev. B 82, 024511 (2010).

13. McMahon, P. L. et al. A fully-programmable 100-spin coherent Ising machine with all-to-all connections. Science 354, 614-617 (2016).

14. Bravyi, S., DiVincenzo, D. P., Oliveira, R. I. \& Terhal, B. M. The complexity of stoquastic local Hamiltonian problems. Quantum Inf. Comput. 8, 0361 (2008).

15. Bravyi, S. \& Terhal, B. Complexity of stoquastic frustrationfree Hamiltonians. SIAM J. Comput. 39, 1462-1485 (2009).

16. Suzuki, M., Miyashita, S. \& Kuroda, A. Monte Carlo simulation of quantum spin systems. I. Prog. Theor. Phys. 58, 1377 (1977).

17. Troyer, M. \& Wiese, U.-J. Computational complexity and fundamental limitations to fermionic quantum Monte Carlo simulations. Phys. Rev. Lett. 94, 170201 (2005).

18. Bravyi, S. Monte Carlo simulation of stoquastic Hamiltonians. Preprint at http:// arxiv.org/pdf/quant-ph/1402.2295 (2014).

19. Heim, B., Rønnow, T. F., Isakov, S. V. \& Troyer, M. Quantum versus classical annealing of Ising spin glasses. Science 348, 215-217 (2015).

20. Bravyi, S. \& Gosset, D. Polynomial-time classical simulation of quantum ferromagnets. Preprint at http://arxiv.org/pdf/quant-ph/1612.05602 (2016).

21. Ohzeki, M. Quantum Monte Carlo simulation of a particular class of nonstoquastic Hamiltonians in quantum annealing. Sci. Rep. 7, 41186 (2017).

22. Farhi, E. \& Harrow, A. W. Quantum supremacy through the quantum approximate optimization algorithm. Preprint at http://arxiv.org/pdf/quant-ph/1602.07674 (2016).

23. Hastings, M. B. \& Freedman, M. H. Obstructions to classically simulating the quantum adiabatic algorithm. Preprint at http://arxiv.org/pdf/quant-ph/ 1302.5733 (2013).

24. Jarret, M., Jordan, S. P. \& Lackey, B. Adiabatic optimization versus diffusion Monte Carlo methods. Phys. Rev. A 94, 042318 (2016).

25. Farhi, E. et al. Quantum adiabatic algorithms, small gaps, and different paths Quantum Inf. Comput. 11, 181-214 (2011).

26. Seoane, B. \& Nishimori, H. Many-body transverse interactions in the quantum annealing of the p-spin ferromagnet. J. Phys. A 45, 435301 (2012).

27. Crosson, E., Farhi, E., Lin, C. Y.-Y., Lin, H.-H. \& Shor, P. Different strategies for optimization using the quantum adiabatic algorithm. Preprint at http://arxiv.org/ pdf/quant-ph/1401.7320 (2014).

28. Seki, Y. \& Nishimori, H. Quantum annealing with antiferromagnetic transverse interactions for the Hopfield model. J. Phys. A 48, 335301 (2015).

29. Zeng, L., Zhang, J. \& Sarovar, M. Schedule path optimization for adiabatic quantum computing and optimization. J. Phys. A 49, 165305 (2016).

30. Hormozi, L., Brown, E. W., Carleo, G. \& Troyer, M. Non-stoquastic Hamiltonians and quantum annealing of Ising spin glass. Preprint at http://arxiv.org/pdf/quant-ph/ 1609.06558 (2016).

31. Nishimori, H. \& Takada, K. Exponential enhancement of the efficiency of quantum annealing by nonstochastic Hamiltonians. Preprint at http://arxiv.org/pdf/quant$\mathrm{ph} / 1609.03785$ (2016).

32. Aharonov, Y. \& Anandan, J. Phase change during a cyclic quantum evolution Phys. Rev. Lett. 58, 1593 (1987).

33. Anandan, J. Non-adiabatic non-abelian geometric phase. Phys. Lett. A 133 171-175 (1988).

34. Zanardi, P. \& Rasetti, M. Holonomic quantum computation. Phys. Lett. A 264 94-99 (1999). 
35. Sjöqvist, E. et al. Non-adiabatic holonomic quantum computation. New J. Phys. 14, 103035 (2012)

36. $\mathrm{Zu}, \mathrm{C}$. et al. Experimental realization of universal geometric quantum gates with solid-state spins. Nature 514, 72-75 (2014).

37. Pirkkalainen, J. M., Solinas, P., Pekola, J. P. \& Möttönen, M. Non-abelian geometric phases in ground-state Josephson devices. Phys. Rev. B 81, 174506 (2010).

38. Kamleitner, I., Solinas, P., Müller, C., Shnirman, A. \& Möttönen, M. Geometric quantum gates with superconducting qubits. Phys. Rev. B 83, 214518 (2011).

39. Makhlin, Y., Schön, G. \& Shnirman, A. Quantum-state engineering with Josephson-junction devices. Rev. Mod. Phys. 73, 357-400 (2001).

40. Berry, M. V. Quantal phase factors accompanying adiabatic changes. Proc. R. Soc. Lond. A Math. Phys. Sci. 392, 45 (1984).

41. Wilczek, F. \& Zee, A. Appearance of gauge structure in simple dynamical systems. Phys. Rev. Lett. 52, 2111-2114 (1984).

42. Boixo, S. et al. Computational multiqubit tunnelling in programmable quantum annealers. Nat. Commun. 7, 10327 (2016).

43. Yan, F. et al. The flux qubit revisited to enhance coherence and reproducibility. Nat. Commun. 7, 12964 (2016).

44. Orlando, T. P. et al. Superconducting persistent-current qubit. Phys. Rev. B 60, 15398-15413 (1999).

45. Weber, S. J. et al. Coherent coupled qubits for quantum annealing. Preprint at http://arxiv.org/pdf/quant-ph/1701.06544 (2017).

46. del Campo, A. Shortcuts to adiabaticity by counterdiabatic driving. Phys. Rev. Lett. 111, 100502 (2013).
47. Takahashi, K. Shortcuts to adiabaticity for quantum annealing. Phys. Rev. A 95, 012309 (2017).

48. Somma, R. D., Nagaj, D. \& Kieferová, M. Quantum speedup by quantum annealing. Phys. Rev. Lett. 109, 050501 (2012).

49. Jordan, S. P., Gosset, D. \& Love, P. J. Quantum-Merlin-Arthur-complete problems for stoquastic Hamiltonians and Markov matrices. Phys. Rev. A 81, 032331 (2010).

(c) Open Access This article is licensed under a Creative Commons Attribution 4.0 International License, which permits use, sharing, adaptation, distribution and reproduction in any medium or format, as long as you give appropriate credit to the original author(s) and the source, provide a link to the Creative Commons license, and indicate if changes were made. The images or other third party material in this article are included in the article's Creative Commons license, unless indicated otherwise in a credit line to the material. If material is not included in the article's Creative Commons license and your intended use is not permitted by statutory regulation or exceeds the permitted use, you will need to obtain permission directly from the copyright holder. To view a copy of this license, visit http://creativecommons. org/licenses/by/4.0/.

c The Author(s) 2017 